\title{
Adjudin delays cellular senescence through Sirt3-mediated attenuation of ROS production
}

\author{
KEYI GENG, NINGZHEN FU, XIAO YANG and WEILIANG XIA \\ State Key Laboratory of Oncogenes and Related Genes, School of Biomedical Engineering, \\ Shanghai Jiao Tong University, Shanghai 200030, P.R. China
}

Received September 25, 2016; Accepted January 6, 2018

DOI: $10.3892 /$ ijmm.2018.3917

\begin{abstract}
Aging, marked by the physical and functional decline in numerous biological processes, is associated with multiple pathologies including cancer, neurodegenerative diseases and cardiocerebral vascular diseases. The accumulation of reactive oxygen species (ROS) production is considered one of the major causes of aging-associated diseases and a major therapeutic target. Hydroxyurea has been widely used for cellular senescence model. The expression level of cell cycle-related protein, ROS production and senescence-associated $\beta$-galactosidase are considered to be markers of cellular senescence. Strategies to slow senescence may be beneficial for various aging-associated diseases. The results of the current study indicated that adjudin, a multi-functional small molecule compound, delayed hydroxyurea-induced senescence in mouse embryo fibroblasts (MEFs). Adjudin reduced the proportion of senescence-associated $\beta$-galactosidase-positive cells and decreased the expression levels of senescence-associated markers, p16 and p21. Mechanistically, adjudin exerted its anti-senescence effect by elevating the expression level of sirtuin 3 (Sirt3), which attenuated ROS production through the regulation of forkhead box O3a and manganese superoxide dismutase expression. Furthermore, by comparing wild-type and Sirt3-knockout MEFs, it was demonstrated that Sirt3 mediated the anti-senescence effect of adjudin. Taken together, the findings indicated that adjudin has anti-aging properties that may be exploited to treat aging-associated diseases.
\end{abstract}

Correspondence to: Dr Weiliang Xia, State Key Laboratory of Oncogenes and Related Genes, School of Biomedical Engineering, Shanghai Jiao Tong University, Room 211, Med-X Research Institute, 1954 Huashan Road, Shanghai 200030, P.R. China

E-mail:wlxia@sjtu.edu.cn

Abbreviations: ROS, reactive oxygen species; HU, hydroxyurea; MEF, mouse embryo fibroblast; SA- $\beta$-gal, senescence-associated $\beta$-galactosidase; Foxo3a, forkhead box O3a; SOD2, manganese superoxide dismutase; CCK- 8 , cell counting kit- 8

Key words: adjudin, cellular senescence, sirtuin 3, reactive oxygen species, manganese superoxide dismutase, forkhead box O3a

\section{Introduction}

Aging causes a progressive loss of tissue function processes (1) marked by numerous common hallmarks, including genome instability, deregulated nutrient sensing, molecular damage, telomere attrition, epigenetic and transcriptional changes, inflammation, cell death and senescence, and metabolic dysfunction $(2,3)$. Cellular senescence refers to a process that imposes permanent proliferative arrest in response to various stressors, emerging as one of the most important contributors to age-associated disease and an attractive target for therapy $(4,5)$. The mechanism underlying senescence remains largely unknown, which makes it challenging to determine the events involved in aging. An enduring potential explanation is oxidative stress. Excessive levels of intracellular reactive oxygen species (ROS) result in age-associated characteristics, including DNA damage, proteins oxidation, lipids degradation and increased ROS production, culminating in significant cellular injury (6). In the nervous system, accumulation of ROS contributes to the age-associated loss of cognitive, sensory and motor function (7).

Sirtuins are a conserved family of deacetylase proteins, which are among the first genes reported to extend lifespan $(8,9)$. Sirtuin 3 (Sirt3), a mitochondrial deacetylase (10), has been reported to regulate major mitochondrial biological processes, including ATP generation, ROS detoxification, nutrient oxidation, mitochondrial dynamics and the unfolded protein response (11-15) by removing acetyl modifications from mitochondrial proteins $(10,16)$ or other acyl modifications and histone crotonylation $(17,18)$. A number of mitochondrial proteins involved in aging process are in an acetylated form (19) and are substrates of Sirt3 (10), suggesting that Sirt3 may mediate a broad spectrum of protection against ROS-induced aging.

Adjudin, formerly termed AF-2364 [1-(2,4-dichlorobenzyl) 1H-indazole-3-carbo-hydrazide] (20), exhibits reversible anti-spermatogenic activity through disruption of adherent junctions of premature sperm to seminiferous epithelium $(21,22)$. We have previously reported that adjudin reduced ischemia-induced neuroinflammation through the nuclear factor- $\kappa \mathrm{B}(\mathrm{NF}-\kappa \mathrm{B})$ pathway (23) and reduced loss of gentamycin-induced rodent cochlear hair cells through the Sirt3-ROS axis (24). The current study aimed to investigate whether adjudin has anti-aging effects and the underlying mechanisms involved. 


\section{Materials and methods}

Reagents and animals. Hydroxyurea (HU) and reactive oxygen species (in dimethyl sulfoxide) were purchased from Sinopharm Chemical Reagent Co., Ltd. (Beijing, China) and Sigma-Aldrich (Merck KGaA, Darmstadt, Germany), respectively. Adjudin was provided by Mary M. Wohlford Laboratory (Population Council, New York, USA). Wild-type (WT) C57BL/129 mice and Sirt3-knockout (KO) C57BL/129 mice were acquired from the Jackson Laboratory (Sacramento, CA, USA). Animal procedures for this research were based on the Institutional Animal Care and Use Committee of Shanghai Jiao Tong University (Shanghai, China).

Cell culture and mouse embryo fibroblast (MEF) isolation. MEFs were isolated from embryonic 13.5 (E13.5) WT or Sirt3-KO mice. This study was approved by the Bioethics Committee of School of Biomedical Engineering, Shanghai Jiao Tong University. The embryos, excluding the head and visceral tissues, were washed with phosphate-buffered saline (PBS), minced with scissor and then transferred into $0.1 \mathrm{mM}$ trypsin $/ 1 \mathrm{mM}$ EDTA solution. After incubation at $37^{\circ} \mathrm{C}$ for $20 \mathrm{~min}$, twice the amount of medium was added and cells were cultured in Dulbecco's modified Eagle's medium (DMEM) containing $10 \%$ fetal bovine serum (FBS; Gibco; Thermo Fisher Scientific, Inc., Waltham, MA, USA) and antibiotics (penicillin, $100 \mathrm{U} / \mathrm{ml} ; 100 \mu \mathrm{g} / \mathrm{ml}$, streptomycin) at $37^{\circ} \mathrm{C}$ in a humidified incubator with $5 \% \mathrm{CO}_{2}$.

Cell viability assay. Cell viability was determined using Cell Counting kit-8 (CCK-8; Dojindo Molecular Technologies, Inc., Kumamoto, Japan). Briefly, $10,000 \mathrm{MEFs}$ were seeded into one well of 96-well plates. Different concentrations of adjudin were tested both with and without hydroxyurea $(24 \mathrm{~h}, 6 \mathrm{mM}$ : 0 , $5,10,20$ and $40 \mu \mathrm{M})$. The cells were pre-treaeted with adjudin for $1 \mathrm{~h}$ and then incubated with/without hydroxyurea for $24 \mathrm{~h}$. Following treatment, CCK-8 solution was added to each well at the dilution of 1:10. After incubation for $\sim 1.5 \mathrm{~h}$, the absorbance at $450 \mathrm{~nm}$ was measured using a microplate reader (Synergy2; BioTek Instruments, Inc., Winooski, VT, USA).

Senescence-associated $\beta$-galactosidase (SA- $\beta$-gal) staining. Cellular senescence was determined by SA- $\beta$-gal staining with senescence-associated $\beta$-galactosidase staining kit (Beyotime Institute of Biotechnology, Haimen, China). Cells were fixed with $4 \%$ paraformaldehyde for $15 \mathrm{~min}$ and washed with PBS three times. Subsequently, cells were incubated overnight at $37^{\circ} \mathrm{C}$ in darkness with the working solution containing $0.05 \mathrm{mg} / \mathrm{ml}$ 5-bromo-4-chloro-3-indolyl-b-d-galactopyranoside (X-gal).

Western blot analysis. Western blotting was performed as previously described (25). Cells were lysed in radioimmunoprecipitation assay lysis buffer (EMD Millipore, Billerica, MA, USA) containing Complete Protease Inhibitor Cocktail (1:100) and $2 \mathrm{mM}$ phenylmethylsulfonyl fluoride. The protein concentration was quantified by bicinchoninic acid protein assay kit (Pierce; Thermo Fisher Scientific, Inc.). Total protein $(30 \mu \mathrm{g})$ was separated by $10 \%$ SDS-PAGE and then transferred to a $0.45 \mu \mathrm{m}$ nitrocellulose membrane (EMD Millipore). Following the incubation with primary antibodies at $4^{\circ} \mathrm{C}$ overnight, the membrane was hybridized with horseradish peroxidase-conjugated secondary antibody (1:5,000 dilution; 111-035-003; Jackson Laboratory) at room temperature for $1 \mathrm{~h}$. Protein signals were visualized by enhanced chemiluminescence detection. The primary antibodies used were as follows: p16 (1:1,000 dilution; ab51243) and p21 (1:1,000 dilution; ab109199; both from Abcam, Shanghai, China); Sirt3 (1:1,000 dilution; 5490S; Cell Signaling Technology, Inc., Danvers, MA, USA); Sirt6 (1:1,000 dilution; ab191385; Abcam); MAPK family antibody [extracellular signal-regulated kinase (ERK), p38, c-Jun N-terminal kinase (JNK); 9926; Cell Signaling Technology, Inc.]; phospho-MAPK family antibody [phospho-ERK, phospho-p38, phospho-JNK; 1:1,000 dilution; Cell Signaling Technology, Inc.)]; manganese superoxide dismutase (SOD2; 1:1,000; Santa Cruz Biotechnology, Inc., Dallas, TX, USA); Akt (1:1,000; ab8805), phospho-Akt (1:1,000; ab38449; both from Abcam); forkhead box O3a (Foxo3a; 1:1,000; 12829; Cell Signaling Technology, Inc.); and $\beta$-tubulin (1:1,000; ab6046; Abcam).

Reverse transcription-quantitative polymerase chain reaction (RT-qPCR). Total RNA was isolated from MEFs with the use of RNAiso Plus (Takara Biotechnology Co., Ltd., Dalian, China), and first strand cDNA was synthesized from $1 \mu \mathrm{g}$ of total RNA using a PrimeScript RT reagent kit (Takara Biotechnology Co., Ltd.). RT-qPCR was performed on an ABI 7900HT (Thermo Fisher Scientific, Inc.) by using SYBR Premix Ex Taq (Takara Biotechnology Co., Ltd.) according to the following protocol: $95^{\circ} \mathrm{C}$ for $30 \mathrm{sec}$; 40 cycles consisting of $95^{\circ} \mathrm{C}$ for $5 \mathrm{sec}$ and $60^{\circ} \mathrm{C}$ for $30 \mathrm{sec}, 95^{\circ} \mathrm{C}$ for $15 \mathrm{sec}$ and $60^{\circ} \mathrm{C}$ for $1 \mathrm{~min}$; and $95^{\circ} \mathrm{C}$ for $15 \mathrm{sec}$. Primers used were as follows: mSirt1 (sense, 5'-TAGTCCTTCCTACCCCAA TTTCC-3' and antisense, 5'-TTGGTCCTTAGCCACTCC TTC-3'); mSirt2 (sense, 5'-GCCTGGGTTCCCAAA AGGAG-3' and antisense, 5'-GAGCGGAAGTCAGGG ATACC-3'); mSirt3 (sense, 5'-ATCCCGGACTTCAGA TCCCC-3' and antisense, 5'-CAACATGAAAAAGGGCTT GGG-3'); mSirt4 (sense, 5'-GTGGAAGAATAAGAATGA GCGGA-3' and antisense, 5'-GGCACAAATAACCCC GAGG-3'); mSirt5 (sense, 5'-CTCCGGGCCGATTCA TTTCC-3' and antisense, 5'-GCGTTCGCAAAACAC TTCCG-3'); mSirt6 (sense, 5'-ATG TCGGTGAATTATGCA GCA-3' and antisense, 5'-GCTGGAGGACTGCCA CATTA-3'); mSirt7 (sense, 5'-AGCATCACCCGTTTG CATGA-3' and antisense, 5'-GGCAGTACGCTCAGT CACAT-3'); mSOD2 (sense, 5'-CAGACCTGCCTTACGACT ATGG-3' and antisense, 5'-CTCGGTGGCGTTGAGATT GTT-3'); m ribosomal protein, large P0 (mRplp0; sense 5'-AGATTCGGGATATGCTGTTGGC-3' and antisense, 5'-TCGGGTCCTAGACCAGTGTTC-3'). RT-qPCR was performed out in triplicate and the results are presented as the $\mathrm{Cq}$ values. The mean $\mathrm{Cq}$ value was calculated, and the $\Delta \mathrm{Cq}$ value was determined as the mean $\mathrm{Cq}$ value for the target gene minus the mean $\mathrm{Cq}$ value for mRplp0. Relative mRNA expression was calculated using the $2^{-\Delta \Delta \mathrm{Cq}}$ method.

ROS assay. Following treatment, cells were washed with PBS. Dicholorofluorescein diacetate (DCF-DA; Beyotime Institute of Biotechnology) was diluted in FBS-free DMEM to $10 \mu \mathrm{M}$ and then added to each well. After incubation for $0.5 \mathrm{~h}$, cells were washed with PBS three times, and the fluorescence was 
A

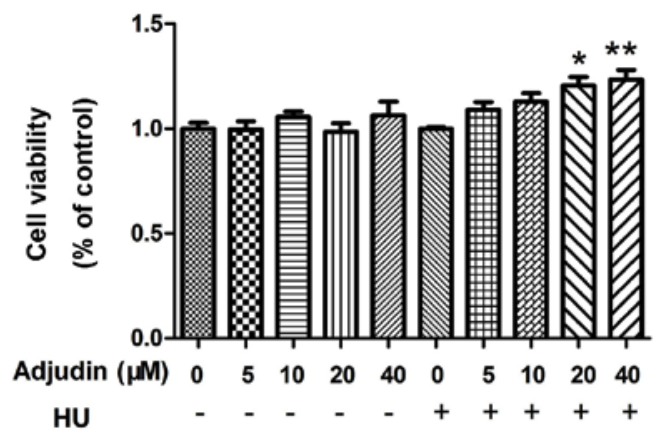

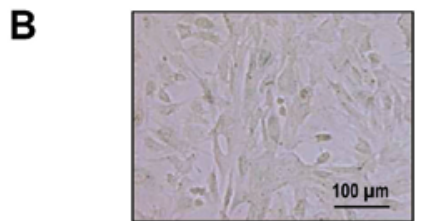

Adjudin $(\mu \mathrm{M}) \quad 0$

$\mathrm{HU}$

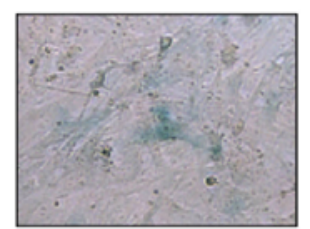

Adjudin ( $\mu \mathrm{M})$

HU

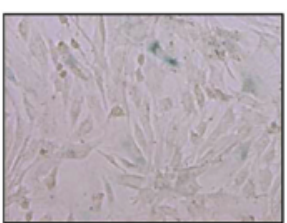

10

-

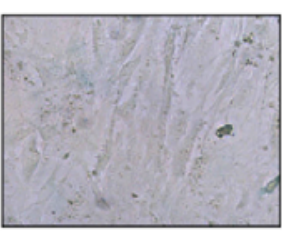

10

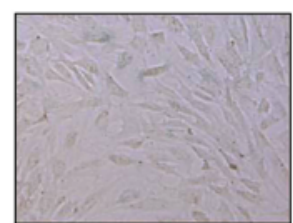

20

-

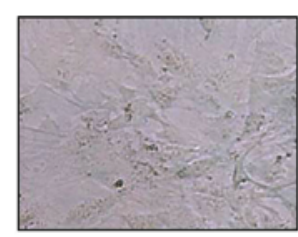

20

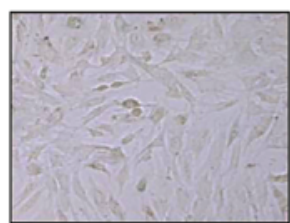

40

-

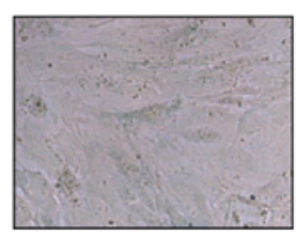

40
C

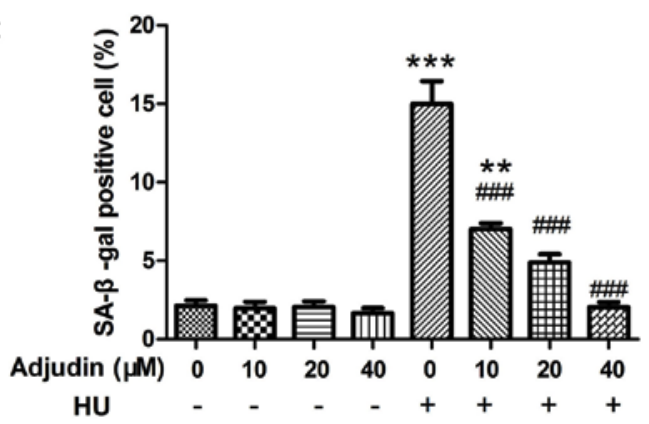

D Adjudin
$\mathrm{HU}$

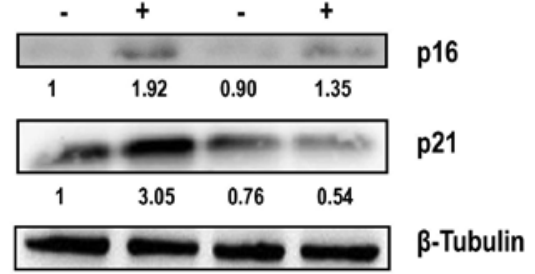

Figure 1. Adjudin attenuates HU-induced cell senescence. MEF cells were pretreated with indicated concentrations of adjudin for $1 \mathrm{~h}$ and then stimulated with $6 \mathrm{mM}$ HU for a 24-h incubation period. (A) MEF cells were pretreated with adjudin at 5, 10, 20 and $40 \mu \mathrm{M}$ in the absence or presence of HU and cell viability was determined by the cell counting kit-8 assay. (B) MEF cells were treated with adjudin at 10, 20 and $40 \mu \mathrm{M}$ in the absence or presence of HU and stained for SA- $\beta$-gal. Scale bar, $100 \mu \mathrm{m}$. (C) Percentage of SA- $\beta$-gal activity positive cells was determined by counting blue cells counting 400 cells/well. (D) Cells were pretreated with adjudin at $40 \mu \mathrm{M}$. Cell lysates were analyzed by immunoblotting with antibodies specific to p16 and p21. $\beta$-tubulin was used as a reference. Data are presented as the mean \pm standard deviation of three independent experiments. ${ }^{*} \mathrm{P}<0.05,{ }^{* *} \mathrm{P}<0.01$ and ${ }^{* * * *} \mathrm{P}<0.001$ vs. control treatment; ${ }^{\# \# \#} \mathrm{P}<0.001$ vs. HU alone treatment. HU, hydroxyurea; MEF, mouse embryo fibroblast; SA- $\beta$-gal, senescence-associated $\beta$-galactosidase.

detected using a FACSCalibur (BD Biosciences, Franklin Lakes, NJ, USA) flow cytometer at an excitation wavelength of $488 \mathrm{~nm}$ and an emission wavelength of $535 \mathrm{~nm}$.

Statistical analysis. Data are presented as the mean \pm standard deviation. Multiple comparisons were analyzed by one-way analysis of variance followed by Tukey's post hoc test. Statistical analyses were performed using GraphPad Prism 5 (GraphPad Software, Inc., La Jolla, CA, USA). P<0.05 was considered to indicate a statistically significant difference.

\section{Results}

Adjudin delays $\mathrm{HU}$-induced cellular senescence. Cells were treated with $\mathrm{HU}$, which inhibits ribonucleotide reductase activity $(26,27)$, to produce an in vitro senescence model. HU-induced senescence has been widely used to mimic cell aging (28-34). MEFs were pretreated with adjudin for $1 \mathrm{~h}$, followed by a 24-h incubation period with $6 \mathrm{mM}$ HU. As presented in Fig. 1A, cell viability following treatment with the indicated concentrations of adjudin was not significantly 


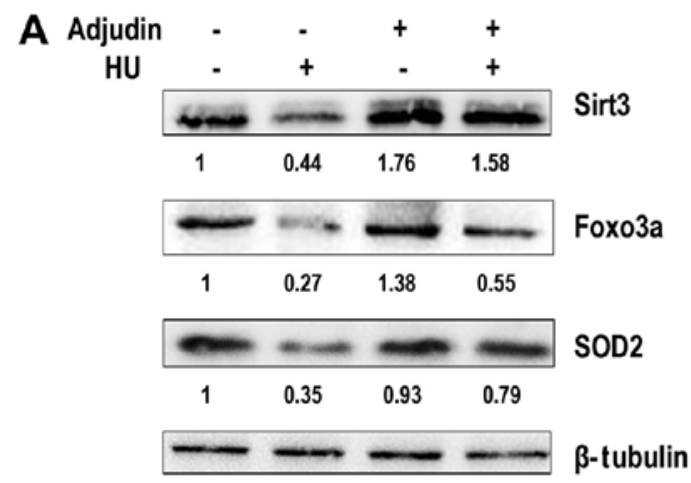

C

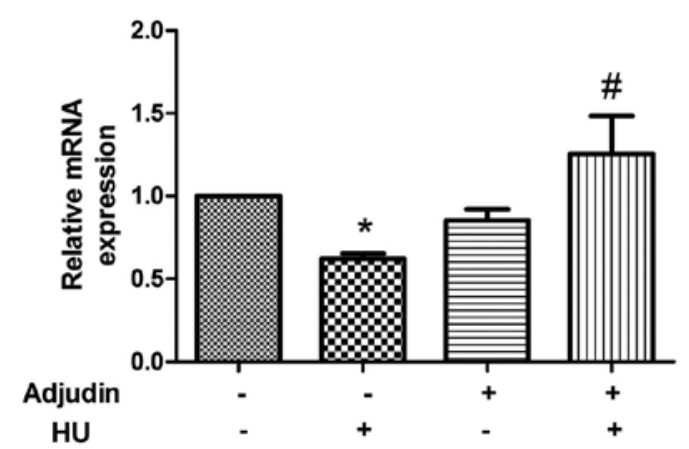

B

Sirt3

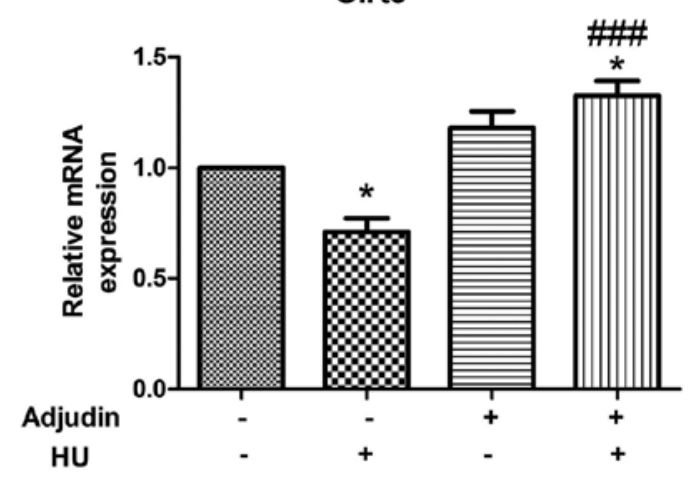

D

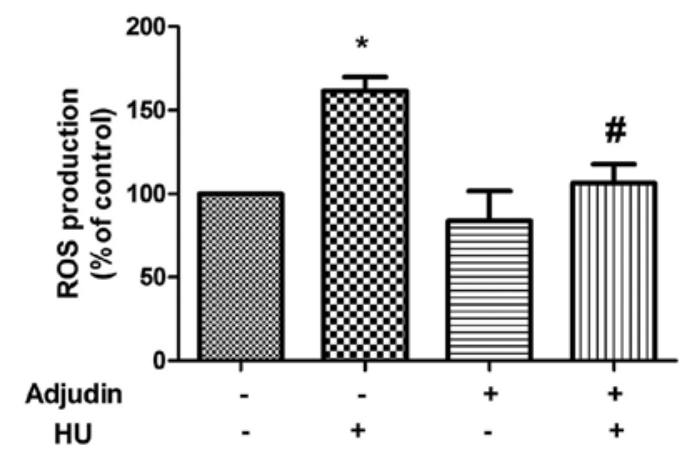

Figure 2. Adjudin upregulates Sirt3 and reduces ROS production. Cells were pretreated with $40 \mu \mathrm{M}$ of adjudin for $1 \mathrm{~h}$ and then stimulated with $6 \mathrm{mM}$ HU for $24 \mathrm{~h}$. (A) Cell lysates were analyzed by immunoblotting with antibodies specific to Sirt3, Foxo3a and SOD2. Equal loading of proteins was estimated by $\beta$-tubulin. (B) Sirt3 and (C) SOD2 mRNA expression was evaluated by quantitative polymerase chain reaction. Data were normalized to ribosomal protein, large P0. (D) Cells were stimulated with HU for $24 \mathrm{~h}$, and the total ROS level was determined using dicholorofluorescein diacetate. Data are presented as the mean \pm standard of three independent experiments and are presented relative to control. ${ }^{*} \mathrm{P}<0.05$ vs. control treatment. ${ }^{\#} \mathrm{P}<0.05$ and ${ }^{\# \# "} \mathrm{P}<0.001$ vs. HU alone treatment. HU, hydroxyurea; Sirt3, sirtuin 3; Foxo3a, forkhead box O3a; SOD2, manganese superoxide dismutase 2; ROS, reactive oxygen species.

different from the control group in the absence of HU, while a slight elevation in cell survival was observed when treated with 20 and $40 \mu \mathrm{M}$ adjudin in the presence of HU.

Subsequently, whether adjudin could affect cellular senescence was determined. The results demonstrated that pretreatment with adjudin $(10,20$ and $40 \mu \mathrm{M})$ for $1 \mathrm{~h}$ reduced the percentage of $\mathrm{HU}$-induced SA- $\beta$-gal-positive cells at a dose-dependent manner (Fig. 1B and C). Furthermore, levels of p16 and p21, two cyclin-dependent kinase inhibitors considered as senescence markers $(35,36)$, were detected by western blot. HU increased the expression of p16 and p21, which was notably decreased by pretreatment with $40 \mu \mathrm{M}$ adjudin compared with HU alone (Fig. 1D).

Adjudin elevates Sirt3 expression and suppresses ROS level. Previous studies have implicated sirtuins as key mediators of caloric restriction, which is known to inhibit senescence (37). The effect of adjudin on sirtuin expression was examined, and adjudin significantly upregulated mRNA levels of Sirt3 and Sirt6, but there was no change in Sirt6 protein level between different groups (data not shown).

Adjudin has been reported to protect cochlear hair cell via Sirt3-ROS pathway (24) and Sirt3 mediates antioxidant defense and metabolic adaptation that greatly influences mammalian lifespan (37), so we speculate whether adjudin delays senescence through Sirt3-mediated inhibition of ROS production. Indeed, Sirt3 protein level exhibited a 56\% decline following $\mathrm{HU}$ treatment. In addition, Foxo3a and SOD2, two target proteins of Sirt3 closely associated with ROS, also presented a 73 and $65 \%$ decline in senescent cells, relatively. However, pretreatment with $40 \mu \mathrm{M}$ adjudin significantly counteracted those changes in the presence of HU in MEFs (Fig. 2A). Similar results were also observed at the mRNA level for Sirt3 and SOD2 (Fig. 2B and C).

Subsequently, it was examined whether adjudin could reduce ROS production by using the DCF-DA method. HU stimulation resulted in accumulation of intracellular ROS, which was alleviated by adjudin treatment (Fig. 2D).

Sirt3 mediates anti-senescence effect of adjudin. To validate the role of Sirt 3 in the anti-senescence effects of adjudin, MEFs from WT and Sirt3-KO mice were isolated. Both WT and Sirt3-KO MEFs exhibited strong SA- $\beta$-gal activity following exposure to HU. Notably, adjudin co-treatment with $\mathrm{HU}$ caused a 30\% reduction in SA- $\beta$-gal-positive cells in Sirt3-KO MEFs compared to a $60 \%$ reduction in SA- $\beta$-gal-positive cells in WT MEFs, indicating that Sirt3 may be involved in the anti-aging property of adjudin (Fig. 3A and B). 

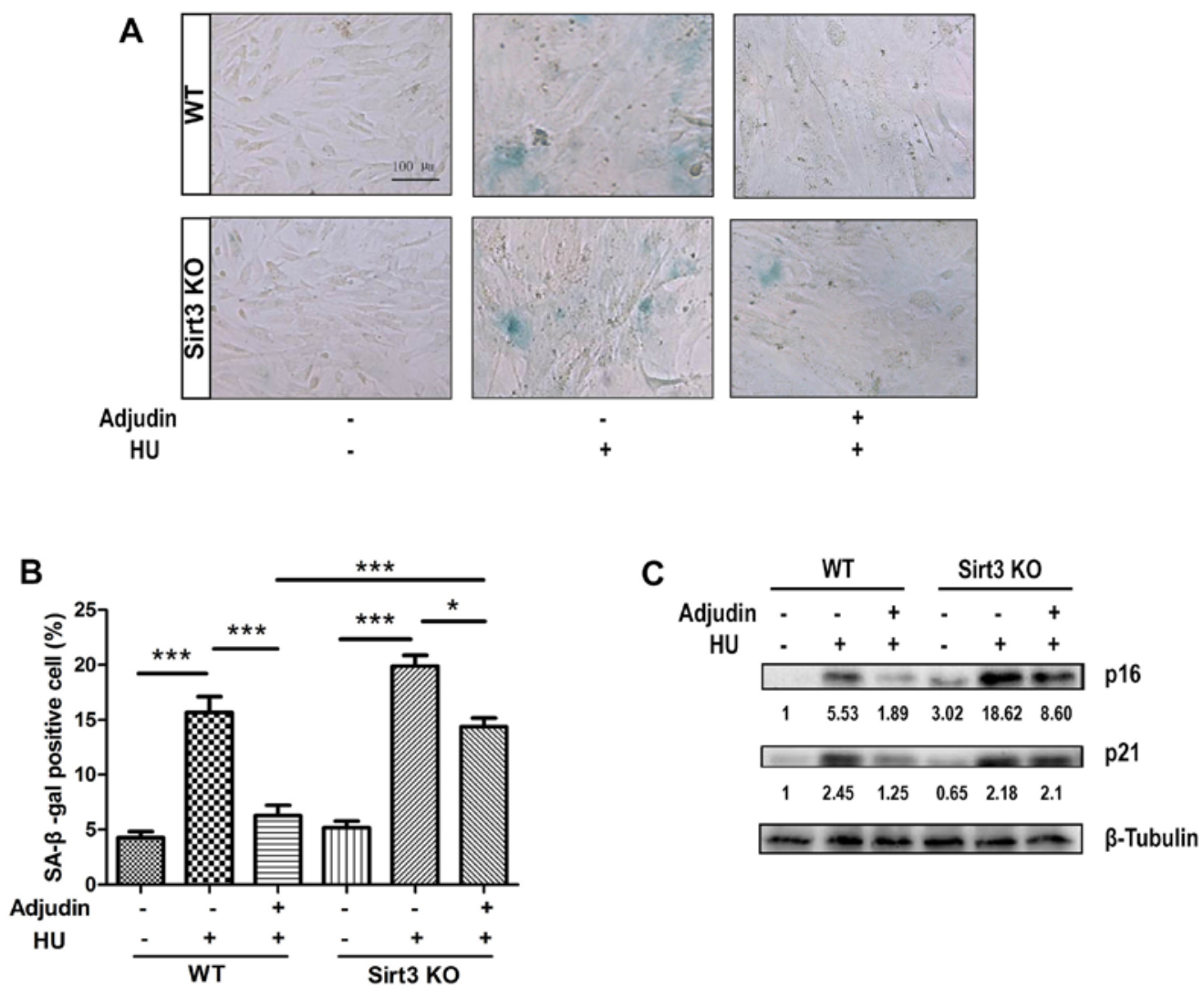

Figure 3. Anti-aging effect of adjudin is mediated by Sirt3. Cells were pretreated with $40 \mu \mathrm{M}$ of adjudin for $1 \mathrm{~h}$ and then stimulated with $6 \mathrm{mM}$ HU for a 24-h incubation period. (A) Cells were stained for SA- $\beta$-gal. Scale bar, $100 \mu \mathrm{m}$. (B) Percentage of SA- $\beta$-gal activity positive cells was determined by counting 400 cells/well. (C) Cell lysates were analyzed by immunoblotting with antibodies specific to 16 and $\mathrm{p} 21$. $\beta$-tubulin was used as a reference for equal loading. Data are presented as the mean \pm standard of three independent experiments. " $\mathrm{P}<0.05$ and ${ }^{* * *} \mathrm{P}<0.001$. WT, wild-type; Sirt 3 , sirtuin; KO knockout; HU, hydroxyurea.
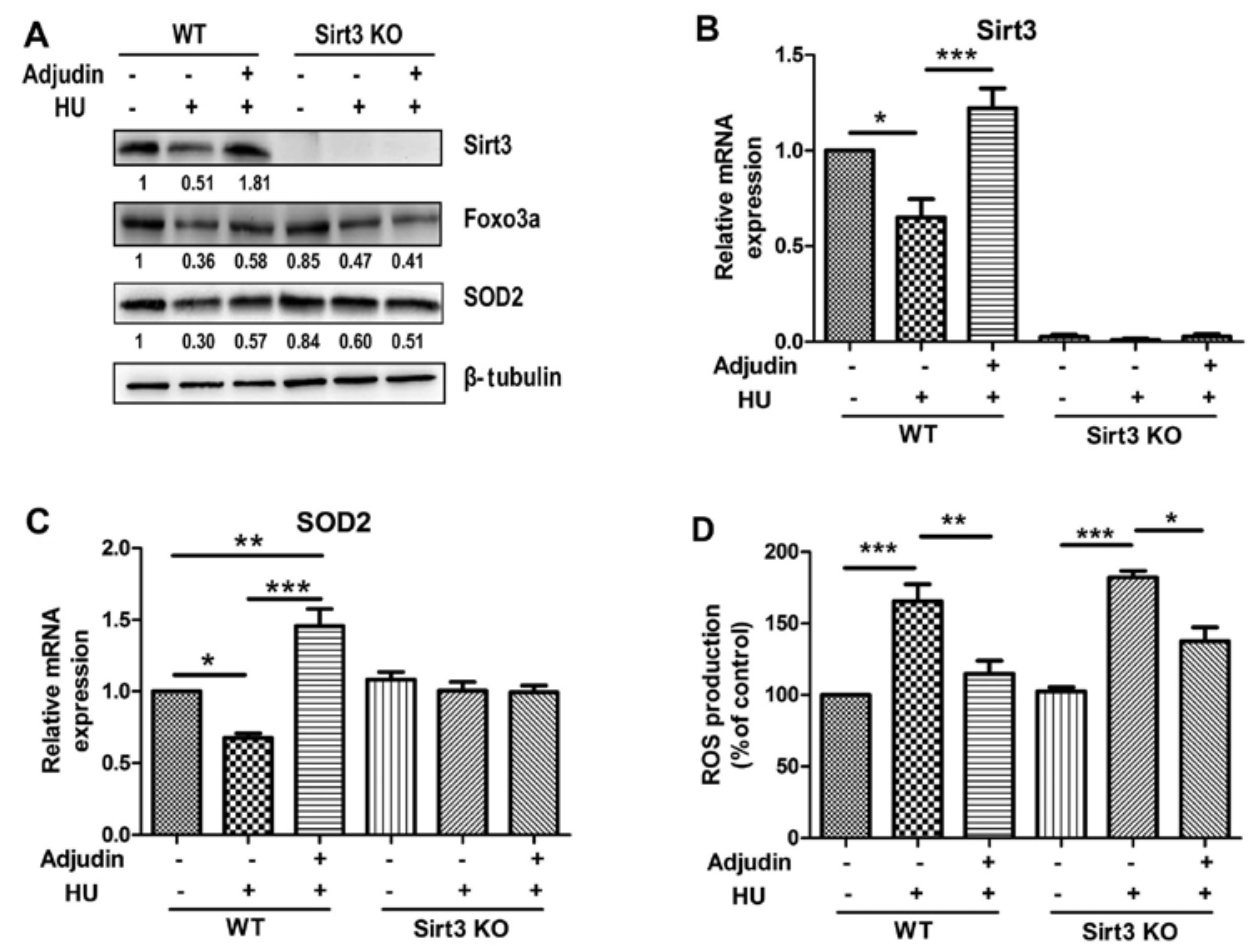

Figure 4. Adjudin exerts anti-aging effects via a Sirt3/ROS pathway. Cells were pretreated with $40 \mu \mathrm{M}$ of adjudin for $1 \mathrm{~h}$ and then stimulated with $6 \mathrm{mM}$ HU for $24 \mathrm{~h}$. (A) Cell lysates were analyzed by immunoblotting with antibodies specific to Sirt3, Foxo3a and SOD2. $\beta$-tubulin was used as internal control for proteins. (B) Sirt3 and (C) SOD2 mRNA expression was evaluated by quantitative polymerase chain reaction. (D) The total ROS level was determined using dicholorofluorescein diacetate. Data were normalized to ribosomal protein large P0. Data are presented as the mean \pm standard deviation of three independent experiments and are presented relative to the control. ${ }^{*} \mathrm{P}<0.05,{ }^{* * *} \mathrm{P}<0.01$ and ${ }^{* * * *} \mathrm{P}<0.001$. WT, wild-type; Sirt3, sirtuin; KO knockout; HU hydroxyurea; Foxo3a, forkhead box O3a; SOD2, manganese superoxide dismutase 2; ROS, reactive oxygen species. 

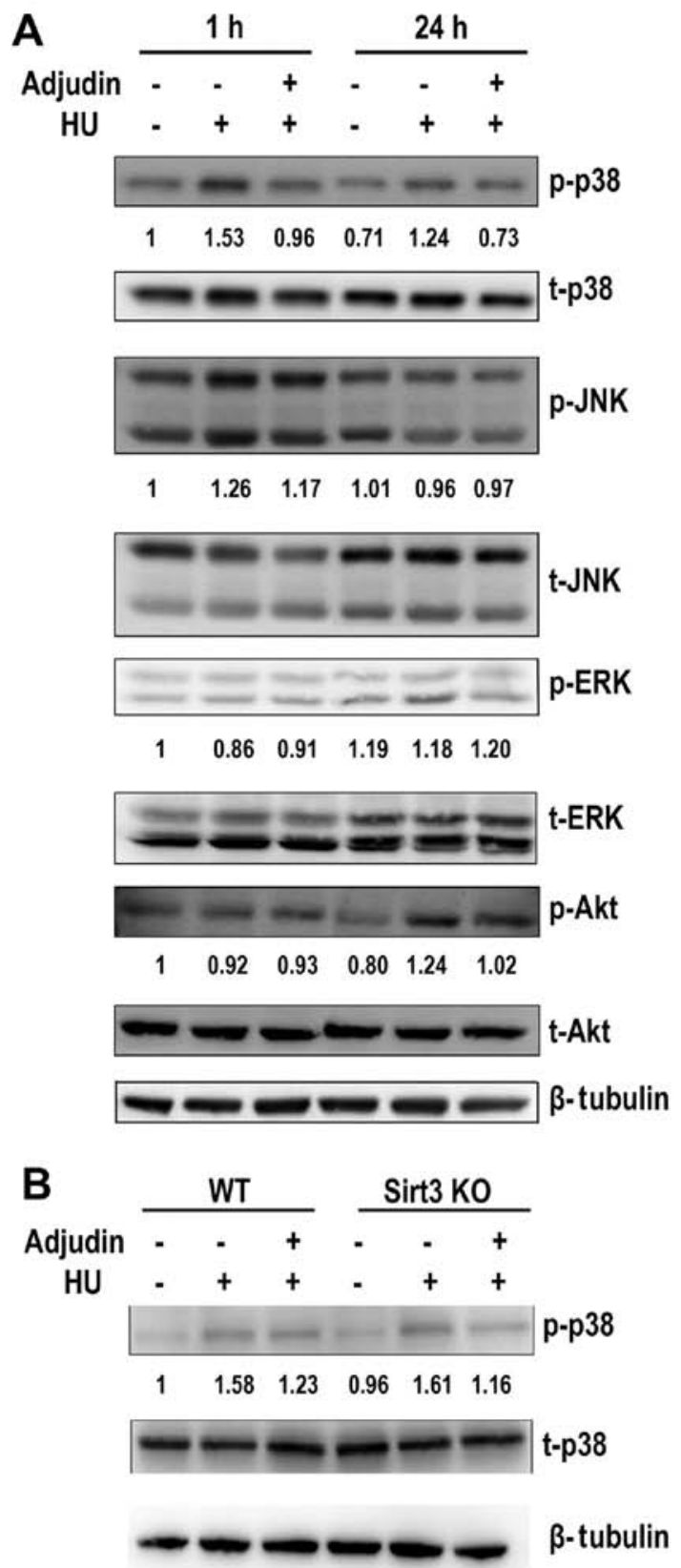

Figure 5. Adjudin suppress the phosphorylation of p38. (A) Cells were treated with $\mathrm{HU}$ for 1 or $24 \mathrm{~h}$. Cell lysates were analyzed by immunoblotting with antibodies specific to p-p38 and p38, p-JNK and JNK, p-Akt and Akt, p-ERK1/2 and ERK1/2, and $\beta$-tubulin. (B) WT and Sirt3 KO cells were treated with HU for $24 \mathrm{~h}$. Western blot analysis of p-p38 and p38 were performed. HU hydroxyurea; p-, phospho; t-, total; JNK, c-Jun N-terminal kinase; ERK, extracellular signal-regulated kinase; WT, wild-type; Sirt3, sirtuin; KO knockout.

In agreement with the SA- $\beta$-gal staining results, p16 and p21 levels were also induced by HU in WT and Sirt3-KO MEFs. Adjudin treatment suppressed the upregulation of p16 and $\mathrm{p} 21$ expression by HU in WT MEFs, while the effect was not as potent in Sirt3-KO MEFs, demonstrating that Sirt3 mediated, at least partially, the anti-senescence effect of adjudin (Fig. 3C).

Adjudin exerts anti-senescence effects via Sirt3/ROS. Sirt3 deficiency prevented adjudin from elevating Foxo3a and SOD2 levels with HU treatment compared with the effects in WT MEFs (Fig. 4A-C). To determine whether Sirt3 influ- ences ROS production, experiments were performed using DCF-DA. Intracellular ROS levels were higher in Sirt3-KO MEFs than in WT MEFs following treatment with HU and adjudin, suggesting that Sirt3 is, at least partially, required for adjudin to perform antioxidant activity (Fig. 4D). This may suggest that other signaling pathways may be involved in this process.

Adjudin decreases phosphorylation of p38 mitogen-activated protein kinase (MAPK). ROS generation activates the MAPK pathway, thus, it was determined whether adjudin affects the MAPK pathway. As presented in Fig. 5A and B, stimulation with $\mathrm{HU}$ induced phosphorylation of $\mathrm{p} 38$ at the early time point $(1 \mathrm{~h})$, which was suppressed by adjudin in WT and Sirt3-KO MEFs, indicating that Sirt3 and p38 play independent roles. However, levels of phosphorylated ERK or JNK were not affected by treatment with adjudin (Fig. 5A). Phosphorylation of Akt was not affected either (Fig. 5A).

\section{Discussion}

The current study identified a new role of adjudin in delaying cellular senescence, demonstrated by reduced SA- $\beta$-gal-positive cells, and p16 and p21 levels. The anti-aging property of adjudin was demonstrated associated with Sirt3-mediated upregulation of Foxo3a and SOD2 expression and attenuation of ROS production, which was validated using Sirt3-KO MEFs.

Mammalian sirtuins (Sirt1-7) are a family of highly conserved $\mathrm{NAD}^{+}$-dependent deacetylases, which are involved in numerous fundamental cellular processes, including metabolic regulation, genomic stability maintenance, DNA repair and stress responses (12). Previous studies have revealed that Sirt3 KO mice spontaneously develop or have accelerated progression of multiple age-associated pathologies, including metabolic syndrome, cancer, cardiovascular diseases, and neurodegenerative diseases (38). Sirt3 has been reported to block aging-associated tissue fibrosis (39) and prevent hearing loss during caloric restriction by stimulating isocitrate dehydrogenase 2 to convert NADP to NADPH in mitochondria, leading to decreased ROS production (40). The results of the current study illustrated that adjudin may upregulate Sirt3 expression in MEFs and in turn counteract premature senescence induced by $\mathrm{HU}$, which supports with the role of Sirt3 in aging and metabolism.

Foxo3a and SOD2 are the two well-established substrates of Sirt3. Foxo3a forms a physical interaction with Sirt3 in mitochondria, and overexpression of Sirt3 increases DNA-binding activity and targeted gene expression of Foxo3a (41). Sirt3 deacetylates Foxo3 at K271 and K290, leading to upregulation of a set of genes that are essential for mitochondrial homeostasis. Consequently, mitochondrial reserve capacity is ensured in response to oxidative damage (42). In addition, Sirt3 deacetylates two important lysine residues on SOD2, thus promoting its antioxidant capacity to reduce oxidative stress damage and extend life span during caloric restriction $(43,44)$. The findings demonstrated that adjudin increased the expression of Foxo3a and SOD2 in WT MEFs; however, this effect was not observed in Sirt3-KO MEFs, indicating Sirt3 is required for the regulation of Foxo3a and SOD2 by adjudin. 
Excessive accumulation of ROS and oxidative stress damage are the main causes of age-associated diseases $(45,46)$. Mitochondrial ROS is linked to the elevation of pro-inflammatory mediators and susceptibility to pathological conditions (47). Thus, the antioxidant property of adjudin in HU-stimulated MEFs was examined. DCF-DA signals revealed lower intracellular ROS levels in adjudin-treated cells, implying contribution to delayed cellular senescence driven by the antioxidant property of adjudin.

In the current study, adjudin also suppressed HU-induced phosphorylation of $\mathrm{p} 38$, and may have roles independent of Sirt3, as lack of Sirt3 in the KO cells did not affect phosphorylation of p38, nor completely abolish the anti-senescence effect of adjudin. It has been reported that p53 restrains constitutive activation of p38 MAPK, preventing the senescence-associated phenotype (48). Another possibility is the MAPK-nuclear factor- $\kappa \mathrm{B}(\mathrm{NF}-\kappa \mathrm{B})$ cascade. Our previous work demonstrated that adjudin inhibited neuroinflammation via attenuation of $\mathrm{NF}-\kappa \mathrm{B}$ signaling pathway (23), which functions as a regulator of redox-sensitive gene expression $(49,50)$.

In conclusion, the results of the current study demonstrated that adjudin upregulated Sirt 3 expression, thus raising Foxo3a and SOD2 levels and reducing ROS production to delay cellular senescence. Further investigation on the role of adjudin in animal models is necessary to confirm that adjudin may be a promising therapeutic option to age-associated diseases.

\section{Acknowledgements}

This study was supported by grants from the Ministry of Science and Technology (grant no. 2013CB945604), the National Key Grant (grant no. 2016YFC0906400), the National Natural Science Foundation, China (grant no. 31270032) and the SJTU funding (grant no. YG2012ZD05).

\section{Competing interests}

The authors declare that they have no competing interests.

\section{References}

1. Flatt T: A new definition of aging? Front Genet 3: 148, 2012.

2. Liao CY and Kennedy BK: SIRT6, oxidative stress, and aging. Cell Res 26: 143-144, 2016.

3. López-Otín C, Blasco MA, Partridge L, Serrano M and Kroemer G: The hallmarks of aging. Cell 153: 1194-1217, 2013.

4. Childs BG, Durik M, Baker DJ and van Deursen JM: Cellular senescence in aging and age-related disease: From mechanisms to therapy. Nat Med 21: 1424-1435, 2015.

5. Campisi J: Aging, cellular senescence, and cancer. Annu Rev Physiol 75: 685-705, 2013.

6. Harman D: The biologic clock: The mitochondria? J Am Geriatr Soc 20: 145-147, 1972 .

7. Lin MT and Beal MF: Mitochondrial dysfunction and oxidative stress in neurodegenerative diseases. Nature 443: 787-795, 2006.

8. Frye RA: Characterization of five human cDNAs with homology to the yeast SIR2 gene: Sir2-like proteins (sirtuins) metabolize NAD and may have protein ADP-ribosyltransferase activity. Biochem Biophys Res Commun 260: 273-279, 1999.

9. Kaeberlein M, McVey M and Guarente L: The SIR2/3/4 complex and SIR2 alone promote longevity in Saccharomyces cerevisiae by two different mechanisms. Genes Dev 13: 2570-2580, 1999.

10. Lombard DB, Alt FW, Cheng HL, Bunkenborg J, Streeper RS, Mostoslavsky R, Kim J, Yancopoulos G, Valenzuela D, Murphy A, et al: Mammalian Sir2 homolog SIRT3 regulates global mitochondrial lysine acetylation. Mol Cell Biol 27: 8807-8814, 2007.
11. Ahn BH, Kim HS, Song S, Lee IH, Liu J, Vassilopoulos A, Deng CX and Finkel T: A role for the mitochondrial deacetylase Sirt3 in regulating energy homeostasis. Proc Natl Acad Sci USA 105: 14447-14452, 2008.

12. Samant SA, Zhang HJ, Hong Z, Pillai VB, Sundaresan NR, Wolfgeher D, Archer SL, Chan DC and Gupta MP: SIRT3 deacetylates and activates OPA1 to regulate mitochondrial dynamics during stress. Mol Cell Biol 34: 807-819, 2014.

13. Hirschey MD, Shimazu T, Goetzman E, Jing E, Schwer B, Lombard DB, Grueter CA, Harris C, Biddinger S, Ilkayeva OR, et al: SIRT3 regulates mitochondrial fatty-acid oxidation by reversible enzyme deacetylation. Nature 464: 121-125, 2010.

14. Jing E, O'Neill BT, Rardin MJ, Kleinridders A, Ilkeyeva OR, Ussar S, Bain JR, Lee KY, Verdin EM, Newgard CB, et al: Sirt3 regulates metabolic flexibility of skeletal muscle through reversible enzymatic deacetylation. Diabetes 62: 3404-3417, 2013.

15. Papa L and Germain D: SirT3 regulates the mitochondrial unfolded protein response. Mol Cell Biol 34: 699-710, 2014.

16. Onyango P, Celic I, McCaffery JM, Boeke JD and Feinberg AP: SIRT3, a human SIR2 homologue, is an NAD-dependent deacetylase localized to mitochondria. Proc Natl Acad Sci USA 99: 13653-13658, 2002.

17. Feldman JL, Baeza J and Denu JM: Activation of the protein deacetylase SIRT6 by long-chain fatty acids and widespread deacylation by mammalian sirtuins. J Biol Chem 288: 31350-31356, 2013.

18. Bao X, Wang Y, Li X, Li XM, Liu Z, Yang T, Wong CF, Zhang J, Hao Q and Li XD: Identification of 'erasers' for lysine crotonylated histone marks using a chemical proteomics approach. eLife: Nov 4, 2014 (Epub ahead of print). doi: 10.7554/eLife.02999.

19. Kim SC, Sprung R, Chen Y, Xu Y, Ball H, Pei J, Cheng T, Kho Y, Xiao H, Xiao L, et al: Substrate and functional diversity of lysine acetylation revealed by a proteomics survey. Mol Cell 23: 607-618, 2006.

20. Floridi A, Paggi MG, D'Atri S, De Martino C, Marcante ML, Silvestrini B and Caputo A: Effect of lonidamine on the energy metabolism of Ehrlich ascites tumor cells. Cancer Res 41: 4661-4666, 1981.

21. Cheng CY, Silvestrini B, Grima J, Mo MY, Zhu LJ, Johansson E, Saso L, Leone MG, Palmery M and Mruk D: Two new male contraceptives exert their effects by depleting germ cells prematurely from the testis. Biol Reprod 65: 449-461, 2001.

22. Xia W and Geng K: A sirtuin activator and an anti-inflammatory molecule-multifaceted roles of adjudin and its potential applications for aging-related diseases. Semin Cell Dev Biol 59: 71-78, 2016.

23. Shao J, Liu T, Xie QR, Zhang T, Yu H, Wang B, Ying W, Mruk DD, Silvestrini B, Cheng CY, et al: Adjudin attenuates lipopolysaccharide (LPS)- and ischemia-induced microglial activation. J Neuroimmunol 254: 83-90, 2013.

24. Quan Y, Xia L, Shao J, Yin S, Cheng CY, Xia W and Gao WQ: Adjudin protects rodent cochlear hair cells against gentamicin ototoxicity via the SIRT3-ROS pathway. Sci Rep 5: 8181, 2015.

25. Xia W, Mruk DD and Cheng CY: C-type natriuretic peptide regulates blood-testis barrier dynamics in adult rat testes. Proc Natl Acad Sci USA 104: 3841-3846, 2007.

26. Krakoff IH, Brown NC and Reichard P: Inhibition of ribonucleoside diphosphate reductase by hydroxyurea. Cancer Res 28 : $1559-1565,1968$.

27. Moore EC and Hurlbert RB: The inhibition of ribonucleoside diphosphate reductase by hydroxyurea, guanazole and pyrazoloimidazole (IMPY). Pharmacol Ther 27: 167-196, 1985.

28. Yeo EJ, Hwang YC, Kang CM, Kim IH, Kim DI, Parka JS, Choy HE, Park WY and Park SC: Senescence-like changes induced by hydroxyurea in human diploid fibroblasts. Exp Gerontol 35: 553-571, 2000.

29. Park MS, Choi JS, Lee W, Yang YJ, Kim J, Lee GJ, Kim SS, Park SH, Kim SC, et al: Pharmacogenomic analysis indicates potential of 1,5-isoquinolinediol as a universal anti-aging agent for different tissues. Oncotarget 6: 17251-17260, 2015.

30. Dong CM, Wang XL, Wang GM, Zhang WJ, Zhu L, Gao S, Yang DJ, Qin Y, Liang QJ, et al: A stress-induced cellular aging model with postnatal neural stem cells. Cell Death Dis 5: e1116, 2014.

31. Min JN, Tian Y, Xiao Y, Wu L, Li L and Chang S: The mINO80 chromatin remodeling complex is required for efficient telomere replication and maintenance of genome stability. Cell Res 23: 1396-1413, 2013.

32. Moiseeva O, Mallette FA, Mukhopadhyay UK, Moores A and Ferbeyre G: DNA damage signaling and p53-dependent senescence after prolonged beta-interferon stimulation. Mol Biol Cell 17: 1583-1592, 2006. 
33. Stewart SA, Ben-Porath I, Carey VJ, O'Connor BF, Hahn WC and Weinberg RA: Erosion of the telomeric single-strand overhang at replicative senescence. Nat Genet 33: 492-496, 2003.

34. Di Micco R, Sulli G, Dobreva M, Liontos M, Botrugno OA, Gargiulo G, dal Zuffo R, Matti V, d'Ario G, et al: Interplay between oncogene-induced DNA damage response and heterochromatin in senescence and cancer. Nat Cell Biol 13: 292-302, 2011.

35. Kim WY and Sharpless NE: The regulation of INK4/ARF in cancer and aging. Cell 127: 265-275, 2006.

36. Herbig U, Jobling WA, Chen BP, Chen DJ and Sedivy JM: Telomere shortening triggers senescence of human cells through a pathway involving ATM, p53, and p21(CIP1), but not p16(INK4a). Mol Cell 14: 501-513, 2004.

37. Baur JA, Ungvari Z, Minor RK, Le Couteur DG and de Cabo R: Are sirtuins viable targets for improving healthspan and lifespan? Nat Rev Drug Discov 11: 443-461, 2012.

38. McDonnell E, Peterson BS, Bomze HM and Hirschey MD: SIRT3 regulates progression and development of diseases of aging. Trends Endocrinol Metab 26: 486-492, 2015.

39. Sundaresan NR, Bindu S, Pillai VB, Samant S, Pan Y, Huang JY, Gupta M, Nagalingam RS, Wolfgeher D, Verdin E, et al: SIRT3 blocks aging-associated tissue fibrosis in mice by deacetylating and activating glycogen synthase kinase $3 \beta$. Mol Cell Biol 36 : 678-692, 2015

40. Someya S, Yu W, Hallows WC, Xu J, Vann JM, Leeuwenburgh C, Tanokura M, Denu JM and Prolla TA: Sirt3 mediates reduction of oxidative damage and prevention of age-related hearing loss under caloric restriction. Cell 143: 802-812, 2010.

41. Jacobs KM, Pennington JD, Bisht KS, Aykin-Burns N, Kim HS, Mishra M, Sun L, Nguyen P, Ahn BH, Leclerc J, et al: SIRT3 interacts with the daf-16 homolog FOXO3a in the mitochondria, as well as increases FOXO3a dependent gene expression. Int J Biol Sci 4: 291-299, 2008.
42. Tseng AH, Shieh SS and Wang DL: SIRT3 deacetylates FOXO3 to protect mitochondria against oxidative damage. Free Radic Biol Med 63: 222-234, 2013.

43. Qiu X, Brown K, Hirschey MD, Verdin E and Chen D: Calorie restriction reduces oxidative stress by SIRT3-mediated SOD2 activation. Cell Metab 12: 662-667, 2010.

44. Tao R, Vassilopoulos A, Parisiadou L, Yan Y and Gius D: Regulation of MnSOD enzymatic activity by Sirt3 connects the mitochondrial acetylome signaling networks to aging and carcinogenesis. Antioxid Redox Signal 20: 1646-1654, 2014.

45. Salminen A, Ojala J, Kaarniranta K and Kauppinen A: Mitochondrial dysfunction and oxidative stress activate inflammasomes: Impact on the aging process and age-related diseases. Cell Mol Life Sci 69: 2999-3013, 2012.

46. Sena LA and Chandel NS: Physiological roles of mitochondrial reactive oxygen species. Mol Cell 48: 158-167, 2012.

47. Li X, Fang P, Mai J, Choi ET, Wang H and Yang XF: Targeting mitochondrial reactive oxygen species as novel therapy for inflammatory diseases and cancers. J Hematol Oncol 6: 19, 2013.

48. Freund A, Patil CK and Campisi J: p38MAPK is a novel DNA damage response-independent regulator of the senescence-associated secretory phenotype. EMBO J 30: 1536-1548, 2011.

49. Bakunina N,Pariante CM and Zunszain PA: Immune mechanisms linked to depression via oxidative stress and neuroprogression. Immunology 144: 365-373, 2015.

50. Hawkes HJ, Karlenius TC and Tonissen KF: Regulation of the human thioredoxin gene promoter and its key substrates: A study of functional and putative regulatory elements. Biochim Biophys Acta 1840: 303-314, 2014 